\title{
High spectral resolution monitoring of Nova V339 Delphini with TIGRE (Corrigendum)
}

I. De Gennaro Aquino ${ }^{1}$, K.-P. Schröder ${ }^{2}$, M. Mittag ${ }^{1}$, U. Wolter ${ }^{1}$, D. Jack², P. Eenens ${ }^{2}$, J. N. González-Pérez ${ }^{1}$, A. Hempelmann ${ }^{1}$, J. H. M. M. Schmitt ${ }^{1}$, P. H. Hauschildt ${ }^{1}$, and G. Rauw ${ }^{3}$

1 Hamburger Sternwarte, Universität Hamburg, Gojenbergsweg 112, 21029 Hamburg, Germany e-mail: ivan.de.gennaro.aquino@hs.uni-hamburg.de

2 Departamento de Astronomía, Universidad de Guanajuato, Apartado Postal 144, 36000 Guanajuato, Mexico

3 Department of Astrophysics, Geophysics and Oceanography, University of Liège, 17 Allée du 6 Août, B5c, 4000 Sart Tilman, Belgium

A\&A 581, A134 (2015), DOI: 10.1051/0004-6361/201525810

\section{ABSTRACT}

Key words. novae, cataclysmic variables - stars: individual: V339 Del - stars: emission-line, Be - instrumentation: spectrographs telescopes - errata, addenda

The tags in Table 2, indicating the positions of the most prominent spectral features in Figs. $2 \mathrm{a}$ and $\mathrm{b}$, were not corrected during the revision process. We include here the corrected table. The lines observed and identified in the spectra and the respective phases remain unchanged.
We kindly thank Patricia A. Whitelock (SAAO/University of Cape Town) for pointing us the error in the table. 
Table 2. Spectral line atlas.

\begin{tabular}{|c|c|c|c|c|c|c|c|c|c|c|c|c|c|c|c|c|c|}
\hline Species $(\AA)$ & A & B & $\mathrm{C}$ & $\mathrm{D}$ & Tag & Species $(\AA)$ & A & B & $\mathrm{C}$ & $\mathrm{D}$ & Tag & Species $(\AA)$ & A & B & $\mathrm{C}$ & $\mathrm{D}$ & Tag \\
\hline H I 3835.4 & $\mathrm{x}$ & $\mathrm{x}$ & $x$ & $\mathrm{x}$ & a.1 & Ti II 4443.8 & $\mathrm{x}$ & & & & & [Fe VI-VII] 5277.0 & & & & $\mathrm{x}$ & \\
\hline Si II 3856.0 & $\mathrm{x}$ & & & & & Ti II 4450.5 & $\mathrm{x}$ & & & & & Fe II 5316.6 & $\mathrm{x}$ & $\mathrm{x}$ & $\mathrm{x}$ & & \\
\hline Si II 3862.6 & $\mathrm{x}$ & & & & & Ti II 4468.5 & $\mathrm{x}$ & & & & & Fe II 5362.8 & $\mathrm{x}$ & & & & \\
\hline H I 3889.1 & $\mathrm{x}$ & $\mathrm{x}$ & $\mathrm{x}$ & $\mathrm{x}$ & a. 2 & He I 4471.5 & & & $\mathrm{x}$ & $\mathrm{x}$ & & Ti II 5381.0 & & $\mathrm{x}$ & & & \\
\hline Ti II 3900.5 & $\mathrm{x}$ & & & & & Mg II 4481.2 & $\mathrm{x}$ & & & & & He II 5412.4 & & & $\mathrm{x}$ & $\mathrm{x}$ & \\
\hline Fe II 3906.0 & $\mathrm{x}$ & & & & & Fe II 4491.4 & $\mathrm{x}$ & & & & & Fe II 5425.0 & & $\mathrm{X}$ & & & \\
\hline Ti II 3913.5 & $\mathrm{x}$ & & & & & Ti II 4501.3 & $\mathrm{x}$ & & & & & Fe II 5532.1 & $\mathrm{X}$ & $\mathrm{x}$ & $\mathrm{X}$ & & \\
\hline He II 3923.0 & & & & $\mathrm{x}$ & & Fe II 4508.3 & $\mathrm{x}$ & & & & & [O I] 5577.3 & & $\mathrm{x}$ & $\mathrm{x}$ & & a. 11 \\
\hline Ca II 3933.7 & $\mathrm{x}$ & $\mathrm{X}$ & $\mathrm{x}$ & & a.3 & N III 4514.9 & & $\mathrm{x}$ & $\mathrm{x}$ & & & Fe I 5660.0 & $\mathrm{X}$ & & & & \\
\hline Ca II 3968.5 & $\mathrm{~b}$ & $\mathrm{~b}$ & $\mathrm{~b}$ & & a. 4 & Fe II 4515.0 & $\mathrm{x}$ & & & & & He I 5875.6 & & & $\mathrm{x}$ & $\mathrm{x}$ & b. 10 \\
\hline H I 3970.1 & $\mathrm{x}$ & $\mathrm{x}$ & $\mathrm{x}$ & $\mathrm{x}$ & a. 4 & Fe II 4522.6 & $\mathrm{x}$ & & & & & $\mathrm{Na}$ I 5889.9 & $\mathrm{X}$ & $\mathrm{x}$ & & & \\
\hline N II 3995.0 & & & $\mathrm{x}$ & & & $\mathrm{Fe}$ II 4534.2 & $\mathrm{x}$ & & & & & 95.9 & $\mathrm{x}$ & $\mathrm{x}$ & & & \\
\hline ?? 4026 & $\mathrm{x}$ & & & & & $\mathrm{Fe}$ II 4549.5 & $\mathrm{x}$ & & & & & N II 5935.0 & & & $\mathrm{x}$ & $\mathrm{x}$ & \\
\hline Ti II 4028.3 & $\mathrm{x}$ & & & & & $\mathrm{Fe}$ II 4555.8 & $\mathrm{x}$ & & & & & C I 6014.0 & $\mathrm{x}$ & $\mathrm{x}$ & & & b. 17 \\
\hline ?? 4048 & $\mathrm{x}$ & & & & & Cr II 4558.6 & $\mathrm{x}$ & & & & & Fe II 6048.1 & $\mathrm{x}$ & & & & \\
\hline Ti II 4053.8 & $\mathrm{x}$ & & & & & Ti II 4572.0 & $\mathrm{x}$ & & & & & [Fe VII] 6087.0 & & & & $\mathrm{x}$ & b. 22 \\
\hline C III 4068.9 & & & $\mathrm{~b}$ & $\mathrm{~b}$ & & Fe II 4583.8 & $\mathrm{x}$ & $\mathrm{x}$ & & & & Fe II 6148.0 & $\mathrm{x}$ & $\mathrm{x}$ & $\mathrm{x}$ & & b. 11 \\
\hline Sr II 4077.7 & $\mathrm{X}$ & & & & & Cr II 4588.2 & $\mathrm{x}$ & & & & & Fe II 6 & $\mathrm{x}$ & & & & \\
\hline H I 4101.7 & $\mathrm{x}$ & $\mathrm{x}$ & $\mathrm{x}$ & $\mathrm{x}$ & a.5 & Cr II 4618.8 & $\mathrm{x}$ & & & & & Fe II 6247.6 & $\mathrm{x}$ & $\mathrm{x}$ & & & b. 12 \\
\hline ?? 4110 & $\mathrm{~b}$ & & & & & $\mathrm{Fe}$ II 4629.3 & $\mathrm{x}$ & $\mathrm{x}$ & $\mathrm{X}$ & & & [O I] 6300.3 & & $\mathrm{x}$ & $\mathrm{x}$ & $\mathrm{x}$ & b. 13 \\
\hline Ti II 4012.4 & $\mathrm{x}$ & & & & & Cr II 4634.1 & $\mathrm{x}$ & & & & & Si II 6347.1 & $\mathrm{X}$ & & & & b. 1 \\
\hline 30.9 & $\mathrm{X}$ & & & & & N III 4638.0 & & & $\mathrm{x}$ & $\mathrm{x}$ & a. 13 & 63.7 & & $\mathrm{x}$ & $\mathrm{x}$ & $\mathrm{X}$ & b. 14 \\
\hline $\mathrm{Fe}$ I 4163.7 & $\mathrm{x}$ & & & & & Fe I 4657.6 & $\mathrm{x}$ & & & & & Si II 6371.4 & $\mathrm{X}$ & & & & b. 2 \\
\hline Fe II 4173.5 & $\mathrm{x}$ & $\mathrm{b}$ & $\mathrm{b}$ & & & Sc II 4670.4 & $\mathrm{x}$ & & & & & Fe II 6456.4 & $\mathrm{x}$ & $\mathrm{x}$ & & & \\
\hline Fe II 4178.9 & $\mathrm{x}$ & $\mathrm{b}$ & $\mathrm{b}$ & & & He II 4685.8 & & & $\mathrm{x}$ & $\mathrm{x}$ & a. 14 & N II 6483.8 & & $\mathrm{x}$ & $\mathrm{x}$ & $\mathrm{x}$ & \\
\hline C III 4186.9 & & & $\mathrm{~b}$ & & & Fe II 4732.9 & $\mathrm{x}$ & $\mathrm{x}$ & & & & H I 6562.7 & $\mathrm{x}$ & $\mathrm{x}$ & $\mathrm{x}$ & $\mathrm{x}$ & b. 3 \\
\hline Sr II 4215.5 & $\mathrm{X}$ & & & & & $? ? 4765$ & $\mathrm{x}$ & & & & & ?? 66 & $\mathrm{x}$ & & & & \\
\hline Fe I 4216.2 & $\mathrm{x}$ & & & & & Fe I 4771.7 & $\mathrm{x}$ & & & & & He I 6678.2 & & & $\mathrm{x}$ & $\mathrm{x}$ & b. 18 \\
\hline Fe II 4233.2 & $\mathrm{x}$ & $\mathrm{x}$ & $\mathrm{x}$ & & & [Fe II] 4772.1 & & $\mathrm{x}$ & $\mathrm{x}$ & & & N I 6722.6 & & $\mathrm{x}$ & $\mathrm{x}$ & & b. 15 \\
\hline Cr II 4242.7 & $\mathrm{x}$ & & & & & Ti II 4805.1 & $\mathrm{x}$ & & & & & C I 6828.1 & $\mathrm{X}$ & $\mathrm{x}$ & & & \\
\hline 46.8 & $\mathrm{X}$ & & & & & Cr II 4824.1 & $\mathrm{x}$ & & & & & $\mathrm{He} \mathrm{I}$ & & & $\mathrm{x}$ & $\mathrm{X}$ & b. 19 \\
\hline C II 4267.2 & & & $\mathrm{x}$ & $\mathrm{X}$ & a.12 & H I 4861.3 & $\mathrm{x}$ & $\mathrm{x}$ & $\mathrm{X}$ & $\mathrm{x}$ & a.7 & C I 7115.0 & $\mathrm{x}$ & $\mathrm{x}$ & $\mathrm{x}$ & & b. 4 \\
\hline Ti II 4290.2 & $\mathrm{x}$ & & & & & Fe II 4923.9 & $\mathrm{x}$ & $\mathrm{x}$ & $\mathrm{x}$ & & a. 8 & C II 7235.0 & & $\mathrm{t}$ & $\mathrm{t}$ & $\mathrm{t}$ & b. 20 \\
\hline Fe II 4296.5 & $\mathrm{x}$ & $\mathrm{b}$ & & & & ?? 4931 & $\mathrm{x}$ & & & & & [O II] 7320.0 & & & $\mathrm{t}$ & $\mathrm{t}$ & b. 21 \\
\hline 03.2 & $\mathrm{x}$ & $\mathrm{b}$ & & & & [O III] 4958.9 & & & $\mathrm{x}$ & $\mathrm{x}$ & & N I 7442.3 & $\mathrm{x}$ & $\mathrm{b}$ & & & \\
\hline Ti II 4315.0 & $\mathrm{x}$ & & & & & [O III] 5006.8 & & & $\mathrm{x}$ & $\mathrm{x}$ & a. 15 & N I 7468.2 & $\mathrm{x}$ & $\mathrm{b}$ & & & \\
\hline Ti II 4321.0 & $\mathrm{x}$ & & & & & Fe II 5018.4 & $\mathrm{x}$ & $\mathrm{x}$ & $\mathrm{x}$ & & a. 9 & O I 7773.0 & $\mathrm{x}$ & $\mathrm{x}$ & $\mathrm{x}$ & & b. 5 \\
\hline $\mathrm{Fe} \mathrm{I} 4$ & $\mathrm{x}$ & & & & & ?? 5053 & $\mathrm{x}$ & & & & & C I 8335.2 & $\mathrm{t}$ & $\mathrm{t}$ & & & b. 16 \\
\hline 40.5 & $\mathrm{x}$ & $\mathrm{x}$ & $\mathrm{x}$ & $\mathrm{b}$ & a.6 & He I 5047.7 & & $\mathrm{x}$ & $\mathrm{x}$ & & & O I 8446.3 & $\mathrm{x}$ & $\mathrm{x}$ & $\mathrm{x}$ & $\mathrm{x}$ & b. 6 \\
\hline $\mathrm{Fe}$ II 4351.8 & $\mathrm{x}$ & $\mathrm{b}$ & & & & Ti II 5129.2 & $\mathrm{x}$ & & & & & Ca II 8498.0 & $\mathrm{x}$ & $\mathrm{X}$ & $\mathrm{x}$ & & b. 7 \\
\hline [O III] 4363.0 & & & $\mathrm{~b}$ & $\mathrm{~b}$ & & [Fe VII] 5158.9 & & & & $\mathrm{x}$ & & Ca II 8542.1 & $\mathrm{x}$ & $\mathrm{X}$ & $\mathrm{x}$ & & b. 8 \\
\hline Sc II 4374.5 & $\mathrm{x}$ & & & & & Fe II 5169.0 & $\mathrm{x}$ & $\mathrm{x}$ & $\mathrm{x}$ & & a. 10 & H I 8598.4 & $\mathrm{x}$ & $\mathrm{x}$ & $\mathrm{x}$ & & \\
\hline Fe II 4385.4 & $\mathrm{x}$ & & & & & Fe II 5197.6 & $\mathrm{x}$ & & & & & N I 8629.2 & $\mathrm{x}$ & & & & \\
\hline Ti II 4395.0 & $\mathrm{x}$ & & & & & [N I] 5199.0 & & $\mathrm{x}$ & $\mathrm{x}$ & & & Ca II 8662.1 & $\mathrm{~b}$ & & & & b. 9 \\
\hline Sc II 4400.4 & $\mathrm{x}$ & & & & & Fe II 5234.6 & $\mathrm{x}$ & $\mathrm{x}$ & $\mathrm{x}$ & & & H I 8665.0 & $\mathrm{~b}$ & $\mathrm{x}$ & $\mathrm{x}$ & & b. 9 \\
\hline Fe II 4416.3 & $\mathrm{x}$ & & & & & Fe II 5276.0 & $\mathrm{x}$ & $\mathrm{x}$ & $\mathrm{x}$ & & & H I 8750.5 & $\mathrm{x}$ & $\mathrm{x}$ & $\mathrm{x}$ & & \\
\hline [Fe II] 4416.8 & & $\mathrm{x}$ & $\mathrm{x}$ & & & & & & & & & & & & & & \\
\hline
\end{tabular}

Notes. The listed lines were observed at least once during the different phases of the nova development. A) Optically thick; B) transition; C) early nebular; D) late nebular; $x$ ) observed within the phase; b) heavily blended; t) strongly affected by telluric lines. The tag added for several lines refers to Figs. 2a and b. The wavelength values displayed are the same as used for the radial velocity plots. The label "??" marks the wavelength for features that are observed as absorption lines in all the spectra of the optically thick phase, for which we could not give a proper identification. 